\title{
Estimation de l'héritabilité de quelques caractères chez le Glaïeul (Gladiolus grandiflorus Hort.)
}

Joseph COHAT

avec la collaboration technique de Corentin Tromeur

I.N.R.A., Station d'Amélioration de la Pomme de terre et des Plantes à bulbes, F 29207 Landerneau

Des plantes de Glaïeul appartenant à 6 clones et aux descendances des 15 croisements entre eux ont été cultivées sous 2 conditions de serre et notées individuellement pour la précocité de floraison, la hauteur de la plante, la longueur de l'épi, le nombre de fleurs, l'esthétique de l'épi et la valeur globale de la plante. Une grande part de la variation observée entre les descendances dans le dispositif expérimental est imputable à l'aptitude générale à la combinaison. L'héritabilité, estimée par la régression parent moyen-descendance, est élevée pour les 4 premiers caractères, très faible pour les 2 derniers. Les conséquences pour la sélection sont discutées.

Mots clés additionnels : Précocité de floraison, hauteur de la plante, longueur de l'épi, nombre de fleurs, valeur esthétique, croisement diallèle, régression parent-descendance, sélection clonale.

Additional key words : Flowering earliness, plant height, spike length, floret number, ornamental value, diallel cross, parent-offspring regression, clonal selection.

\section{INTRODUCTION}

Le glaïeul hybride à grandes fleurs (Gladiolus grandiflorus Hort.) est une espèce à multiplication végétative, allogame, tétraploïde $(4 \times=60)$ et fortement hétérozygote. Il est issu d'une succession de croisements interspécifiques complexes (OHRI \& KHOSHOO, 1985). Le schéma de sélection utilisé chez cette espèce est simple : on recherche, dans les descendances de croisements, les individus exceptionnels regroupant le maximum de caractères favorables, la multiplication végétative permettant l'exploitation directe d'un génotype quel que soit son degré d'hétérozygotie. En l'absence de connaissances sur les mécanismes de transmission héréditaire des caractères, le choix des géniteurs reste empirique; il est basé sur une bonne connaissance des phénotypes des cultivars et sur l'expérience du sélectionneur. L'existence d'une forte interaction génotype-milieu vient compliquer la situation.
L'objet du présent travail est de mieux connaître le mode de transmission de quelques caractères pris en compte dans un programme d'amélioration, afin de rendre moins empirique le choix des géniteurs. Six caractères ont été retenus: précocité de floraison, hauteur de la plante et longueur de l'épi en début de floraison, nombre de fleurs par épi, esthétique de l'inflorescence et valeur globale de la plante. Si les 4 premiers caractères sont faciles à noter, les 2 derniers, par contre, sont délicats à juger car complexes et à appréciation subjective. L'héritabilité de ces 6 caractères a été estimée en utilisant la régression parent moyen-descendance dans un dispositif faisant intervenir 6 clones parentaux et les descendances des 15 croisements réalisés entre eux et étudiés sous 2 conditions de culture. Le coefficient de régression, qui caractérise le degré de ressemblance entre enfants et parent moyen, est considéré comme une estimation de l'héritabilité au sens étroit (SIMMONDS, 1979 ; FERNANDEZ \& MILLER, 1985). 


\section{MATÉRIEL ET MÉTHODES}

Afin de disposer simultanément des parents et des descendances sous forme d'un matériel homogène, différentes opérations ont été réalisées : elles sont résumées ci-après.

\section{Année 1983}

Choix de 6 génotypes à caractéristiques physiologiques et morphologiques différentes parmi les clones utilisés dans le programme d'amélioration en cours. L'objectif principal de ce programme est la recherche de génotypes adaptés à la production précoce de fleurs coupées sous abri. La généalogie des génotypes retenus est donnée en Annexe 1. Réalisation des croisements entre ces 6 génotypes. Compte tenu des écarts importants à la floraison, les croisements n'ont été réalisés que dans un seul sens, les variétés tardives n'étant utilisées que comme mères.

\section{Année 1984}

Semis des graines des 15 familles et plantation de caïeux des 6 parents.

\section{Année 1985}

Plantation des bulbes de petit calibre provenant des semis et des cultures de caïeux. Les conditions climatiques très défavorables de l'été ont limité le grossissement des cormus et $n$ 'ont pas permis dans certains cas de disposer des effectifs prévus malgré un nombre de bulbes plantés largement supérieur aux besoins. Récolte, nettoyage, calibrage des cormus. Préparation thermique à $20^{\circ} \mathrm{C}$ durant 6 semaines avant plantation.

\section{Année 1986}

Plantation le 15 janvier dans une serre dont la température était maintenue au minimum à $8^{\circ}-10^{\circ} \mathrm{C}$ (dénommée serre chaude) de bulbes de calibre homogène (14 à $16 \mathrm{~cm}$ de circonférence). Plantation le 29 janvier dans une seconde serre où la température pouvait descendre jusqu'à $3^{\circ}-4^{\circ} \mathrm{C}$ (dénommée serre froide) de bulbes de calibre 12 à $14 \mathrm{~cm}$ de circonférence. Sous les 2 conditions, l'aération se déclenchait dès que la température atteignait $20^{\circ} \mathrm{C}$. La densité était de 64 plantes au $\mathrm{m}^{2}$ planté, une seule hampe florale étant conservée par plante. Dans chaque serre, les 21 lots de bulbes d'effectifs inégaux (14 à 140) étaient disposés en essai bloc à 2 répétitions. Chaque essai était entouré par un cultivar commun afin de limiter l'effet de bordure dû à la lumière. Ce dispositif a per- mis également de contrôler l'homogénéité des conditions de culture.

Sur chaque plante, diverses notations ont été effectuées : date de floraison (stade défini par PAULIN, 1958) transformée ensuite en nombre de jours s'écoulant entre la plantation et la floraison, hauteur de la plante et longueur de l'épi en début de floraison, nombre de fleurs par épi, valeur ornementale de l'épi et valeur globale de la plante. Les plantes des clones parentaux n'ont pas été jugées individuellement pour leurs qualités ornementales. La valeur des 2 derniers caractères est exprimée par une note variant de 0 (nul) à 5 (excellent). Cette note intègre les appréciations plus ou moins subjectives portées sur de nombreux caractères relatifs à la couleur, la pureté du coloris, la forme et la disposition des fleurs, l'harmonie de l'inflorescence, l'équilibre et l'attrait de la plante. Bien souvent, c'est le caractère le plus mal noté qui est prioritaire pour l'attribution de la note d'ensemble. Les notations de ces caractères sont délicates et subjectives, elles ont toujours été effectuées par les mêmes personnes.

Pour chaque caractère, une analyse de variance a été réalisée pour les valeurs propres des clones (décomposition en effets serre, répétition par serre, clone, interaction clone $\times$ serre), puis pour les valeurs en croisement (décomposition en effets serre, répétition par serre, aptitude générale à la combinaison, aptitude spécifique à la combinaison).

Les coefficients de régression parent-descendance pour chaque caractère et pour chaque condition de culture ont été calculés à partir des 15 couples parent moyen-moyenne de la descendance.

$$
\mathrm{b}=\frac{\text { cov. parent moyen-descendance }}{\text { variance parent moyen }}
$$

En l'absence de certitude sur la normalité des distributions des mesures, le coefficient de corrélation de rang $r_{s}$ de SPEARMAN (SNEDECOR \& COCHRAN, 1957) a également été calculé pour vérifier l'information recueillie par la méthode de la régression.

\section{RÉSULTATS}

\section{A. Comportement des clones parentaux et des descen- dances}

Les données du tableau 1 qui regroupe les valeurs des moyennes et des écarts-types des mesures des dif-

TABLEAU 1

Moyennes $(\bar{x})$ et écarts-types (s) des mesures des caractères notés chez les parents en serre froide.

Means $(\bar{x})$ and standard deviations (s) of parent characters in cold greenhouse.

\begin{tabular}{|c|c|c|c|c|c|c|c|c|c|c|c|}
\hline \multirow{3}{*}{ 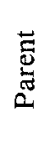 } & \multirow{3}{*}{ 密兽 } & \multirow{2}{*}{\multicolumn{2}{|c|}{$\begin{array}{l}\text { Délai plantation } \\
\text { floraison (jours) }\end{array}$}} & \multirow{2}{*}{\multicolumn{2}{|c|}{$\begin{array}{l}\text { Hauteur } \\
\quad(\mathrm{cm})\end{array}$}} & \multirow{2}{*}{\multicolumn{2}{|c|}{$\begin{array}{l}\text { Longueur } \\
\text { épi }(\mathrm{cm})\end{array}$}} & \multirow{2}{*}{\multicolumn{2}{|c|}{$\begin{array}{l}\text { Nombre de } \\
\text { fleurs }\end{array}$}} & \multirow{2}{*}{\multicolumn{2}{|c|}{$\begin{array}{l}\text { Esthétique } \\
\text { épi globale }\end{array}$}} \\
\hline & & & & & & & & & & & \\
\hline & & $\bar{x}$ & $\mathrm{~s}$ & $\bar{x}$ & $s$ & $\bar{x}$ & $\mathrm{~s}$ & $\bar{x}$ & $\mathrm{~s}$ & $\bar{x}$ & $\bar{x}$ \\
\hline 1 & 35 & 131,3 & 5,8 & 156,8 & 13,1 & 55,1 & 7,0 & 17,1 & 2,1 & 2,75 & 2,75 \\
\hline 2 & 21 & 134,7 & 4,2 & 126,7 & 8,9 & 39,3 & 4,7 & 17,1 & 1,7 & 3,0 & 2,5 \\
\hline 3 & 35 & 113,0 & 3,5 & 120,1 & 7,3 & 44,3 & 6,3 & 12,8 & 1,6 & 3,0 & 3,0 \\
\hline 4 & 35 & 103,5 & 5,5 & 124,7 & 9,9 & 44,3 & 6,1 & 11,4 & 1,6 & 2,25 & 2,5 \\
\hline 5 & 21 & 121,0 & 3,0 & 133,9 & 7,0 & 44,6 & 4,6 & 13,9 & 1,6 & 2,75 & 3,0 \\
\hline 6 & 35 & 120,4 & 4,3 & 146,6 & 11,5 & 52,3 & 8,2 & 14,1 & 2,0 & 2,5 & 2,75 \\
\hline
\end{tabular}


férents caractères notés en serre froide (plantation du 29 janvier) chez les parents, illustrent les différences physiologiques et morphologiques qui existent entre eux. Par exemple, il s'écoule 31 jours entre la floraison du clone le plus précoce (parent 4 ) et celle du plus tardif (parent 2), la hauteur de la plante varie de 120 à $157 \mathrm{~cm}$, le nombre de fleurs par épi de 11,4 à 17,1. Par contre, l'esthétique de l'inflorescence et la valeur globale, dont la note varie respectivement de 2,25 à 3,00 et de 2,50 à 3,00 sont beaucoup moins variables. Les observations faites en serre chaude sont similaires.

L'analyse de variance pour les valeurs propres des clones conduit au tableau 2 qui fait apparaître un fort effet serre et un fort effet clone pour la majorité des caractères.

Le tableau 3 présente les moyennes et écarts-type des mesures effectuées en serre froide sur les descendances. Rappelons que chaque descendance est constituée d'une population de génotypes, tous différents les uns des autres, et représentés par un seul individu. Les écarts maximum observés entre les moyennes des descendances sont en général moins importants que ceux observés chez les parents : par exemple 15,6 jours contre 31,2 pour la précocité de floraison, $31,2 \mathrm{~cm}$ contre 36,7 pour la hauteur de la plante. On observe une diminution très nette des moyennes des notes de l'esthétique de l'inflorescence et de celles de la valeur globale par rapport aux notes des parents et une faible variation entre descendances pour ces 2 caractères. La variation intradescendance est plus élevée que celle observée chez les clones parentaux ; ceci est logique, car outre la variation due au milieu, elle comprend une composante génétique qui est absente chez les parents.

Les coefficients de corrélation phénotypique entre 4 caractères ont été calculés pour chaque condition de culture pour les parents d'une part (tabl. 4) et pour les 15 descendances d'autre part (tabl. 5). Il en ressort que certains caractères sont significativement corrélés :

- nombre de jours entre la plantation et la floraison et le nombre de fleurs par épi ;

- hauteur de la plante et longueur de l'épi ;

- hauteur de la plante et nombre de fleurs par épi, mais seulement dans le cas des descendances.

La première corrélation indique que des descendances à floraison précoce ont tendance à avoir en moyenne un faible nombre de fleurs par épi. Pour savoir si cette corrélation reste valable à l'intérieur d'une descendance, le coefficient de corrélation entre le nombre de jours s'écoulant entre la plantation et la floraison et le nombre de fleurs par épi a été calculé chez les 8 descendances qui fleurissent le plus tôt sous les 2 conditions de culture (tabl. 6). Ce coefficient n'est significativement différent de zéro que dans un

TABLEAU 2

Analyse de variance pour 6 caractères des valeurs propres des clones parentaux. Signification statistique (Si - x : significatif à $5 \%-x x$ à $1 \%-x x x$ à $1 \% \% o$ ) et contribution à la variation générale $(\%$ SCE). Analvsis of variance for 6 characters of parental values. Levels of significance (Si - $x: 5 \%$ level - $x x: 1 \%$ level $-x x x: 0.1 \%$ level) and contribution to general variation (\% SCE).

\begin{tabular}{|c|c|c|c|c|c|c|c|c|c|c|c|c|}
\hline \multirow[b]{2}{*}{ Source de variation } & \multicolumn{2}{|c|}{$\begin{array}{l}\text { Délai plantation } \\
\text { floraison }\end{array}$} & \multicolumn{2}{|c|}{$\begin{array}{l}\text { Hauteur } \\
\text { plante }\end{array}$} & \multicolumn{2}{|c|}{$\begin{array}{l}\text { Longueur } \\
\text { épi }\end{array}$} & \multicolumn{2}{|c|}{$\begin{array}{l}\text { Nombre de } \\
\text { fleurs }\end{array}$} & \multicolumn{2}{|c|}{$\begin{array}{l}\text { Esthétique } \\
\text { épi }\end{array}$} & \multicolumn{2}{|c|}{$\begin{array}{l}\text { Valeur } \\
\text { globale }\end{array}$} \\
\hline & $\mathrm{Si}$ & $\% \mathrm{SCE}$ & $\mathrm{Si}$ & $\%$ SCE & $\mathrm{Si}$ & $\%$ SCE & $\mathrm{Si}$ & $\% \mathrm{SCE}$ & $\mathrm{Si}$ & $\% \mathrm{SCE}$ & $\mathrm{Si}$ & $\%$ SCE \\
\hline Serre & $\mathrm{xx}$ & 1,6 & $\mathrm{xxx}$ & 8,3 & & 2,2 & & 0,5 & $\mathrm{xx}$ & 21,1 & $\mathrm{xx}$ & 30,7 \\
\hline Répétition par serre & & 0,3 & & 0,7 & & 1,1 & & 0,4 & & 0,5 & & 1,8 \\
\hline Clone & $\mathrm{xxx}$ & 96,9 & $\mathrm{xxx}$ & 86,5 & $x x x$ & 84,6 & $\mathrm{xxx}$ & 92,4 & $\mathrm{x}$ & 38,9 & & 29,2 \\
\hline Interaction clone $x$ serre & & 0,4 & & 1,6 & & 6,6 & $\mathrm{xx}$ & 5,0 & & 24,3 & & 20,5 \\
\hline
\end{tabular}

TABLEAU 3

Moyennes $(\bar{x})$ et écarts-types (s) des mesures des caractères notés chez les descendances en serre froide.

Means $(\bar{x})$ and standard deviations of progeny characters in cold greenhouse.

\begin{tabular}{|c|c|c|c|c|c|c|c|c|c|c|c|c|c|}
\hline \multirow{2}{*}{ 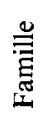 } & \multirow{2}{*}{ 莺总总 } & \multicolumn{2}{|c|}{$\begin{array}{l}\text { Délai plantation } \\
\text { floraison (jours) }\end{array}$} & \multicolumn{2}{|c|}{$\begin{array}{l}\text { Hauteur } \\
\quad(\mathrm{cm})\end{array}$} & \multicolumn{2}{|c|}{$\begin{array}{l}\text { Longueur épi } \\
\qquad(\mathrm{cm})\end{array}$} & \multicolumn{2}{|c|}{$\begin{array}{l}\text { Nombre de } \\
\text { fleurs }\end{array}$} & \multicolumn{2}{|c|}{$\begin{array}{c}\text { Esthétique } \\
\text { épi }\end{array}$} & \multicolumn{2}{|c|}{$\begin{array}{c}\text { Esthétique } \\
\text { globale }\end{array}$} \\
\hline & & $\bar{x}$ & s & $\bar{x}$ & $\mathbf{s}$ & $\bar{x}$ & $\mathrm{~s}$ & $\overline{\mathrm{X}}$ & $s$ & $\bar{x}$ & $\mathrm{~s}$ & $\bar{x}$ & $\mathrm{~s}$ \\
\hline 12 & 70 & 131,8 & 5,3 & 147,4 & 13,6 & 49,1 & 6,8 & 16,8 & 1,9 & 1,42 & 0,41 & 1,59 & 0,39 \\
\hline 13 & 35 & 124,6 & 6,1 & 141,1 & 14,0 & 49,9 & 8,3 & 14,6 & 2,1 & 1,48 & 0,49 & 1,63 & 0,43 \\
\hline 14 & 21 & 120,7 & 5,0 & 138,6 & 16,0 & 49,9 & 10,5 & 13,5 & 2,6 & 1,41 & 0,25 & 1,57 & 0,30 \\
\hline 15 & 49 & 130,7 & 5,5 & 143,7 & 16,5 & 48,1 & 8,7 & 15,9 & 1,8 & 1,62 & 0,40 & 1,72 & 0,36 \\
\hline 16 & 70 & 127,3 & 5,4 & 151,3 & 14,6 & 52,1 & 8,5 & 15,0 & 2,2 & 1,63 & 0,41 & 1,73 & 0,35 \\
\hline 23 & 70 & 126,1 & 7,2 & 133,7 & 18,0 & 47,9 & 9,5 & 15,8 & 3,6 & 1,32 & 0,45 & 1,52 & 0,36 \\
\hline 24 & 70 & 122,2 & 7,0 & 141,0 & 16,6 & 49,2 & 8,6 & 14,1 & 2,3 & 1,41 & 0,32 & 1,56 & 0,34 \\
\hline 25 & 70 & 132,8 & 6,1 & 139,1 & 15,3 & 46,5 & 8,4 & 14,6 & 2,5 & 1,57 & 0,43 & 1,68 & 0,43 \\
\hline 26 & 28 & 126,9 & 5,1 & 142,4 & 13,8 & 51,4 & 7,6 & 15,6 & 2,0 & 1,74 & 0,39 & 1,76 & 0,40 \\
\hline 34 & 14 & 117,2 & 8,8 & 125,8 & 16,7 & 42,7 & 10,0 & 11,4 & 3,0 & 1,61 & 0,37 & 1,35 & 0,44 \\
\hline 35 & 14 & 120,9 & 5,4 & 120,1 & 19,1 & 40,6 & 10,8 & 12,9 & 2,8 & 1,38 & 0,52 & 1,55 & 0,46 \\
\hline 36 & 42 & 121,5 & 7,0 & 141,8 & 15,2 & 53,3 & 10,2 & 14,7 & 2,4 & 1,65 & 0,52 & 1,81 & 0,44 \\
\hline 45 & 56 & 117,7 & 5,8 & 135,9 & 15,3 & 46,8 & 8,8 & 12,1 & 2,0 & 1,41 & 0,35 & 1,62 & 0,35 \\
\hline 46 & 35 & 117,4 & 6,7 & 141,5 & 16,8 & 51,9 & 9,7 & 13,6 & 2,3 & 1,57 & 0,50 & 1,74 & 0,49 \\
\hline 56 & 70 & 123,8 & 5,7 & 143,4 & 16,2 & 50,7 & 9,1 & 13,9 & 2,1 & 1,83 & 0,53 & 1,96 & 0,48 \\
\hline
\end{tabular}


TABLEAU 4

Coefficients de corrélation entre divers caractères chez les parents sous 2 conditions de culture. Signification statistique:

NS : Non significatif

$x$ : Significatif au seuil $5 \%$

$x x$ : Significatif au seuil $1 \%$.

Correlation coefficients between parent characters under 2 cultural conditions.

\begin{tabular}{|c|c|c|c|c|}
\hline $\begin{array}{l}\text { Serre } \\
\text { chaude }\end{array}$ & $\begin{array}{c}\text { Délai } \\
\text { plantation } \\
\text { floraison }\end{array}$ & Hauteur & $\begin{array}{l}\text { Longueur } \\
\text { épi }\end{array}$ & $\begin{array}{l}\text { Nombre } \\
\text { de fleurs }\end{array}$ \\
\hline $\begin{array}{l}\text { Délai plantation } \\
\text { floraison }\end{array}$ & & $\begin{array}{r}0,492 \\
\text { NS }\end{array}$ & $\begin{array}{r}0,126 \\
\text { NS }\end{array}$ & $\begin{array}{r}0,981 \\
\times x\end{array}$ \\
\hline Hauteur & $\begin{array}{r}0,606 \\
\text { NS }\end{array}$ & & $\begin{array}{c}0,893 \\
x\end{array}$ & $\begin{array}{r}0,540 \\
\text { NS }\end{array}$ \\
\hline Longueur épi & $\begin{array}{r}0,584 \\
\text { NS }\end{array}$ & $\begin{array}{c}0,906 \\
x\end{array}$ & & $\begin{array}{r}0,200 \\
\text { NS }\end{array}$ \\
\hline Nombre de fleurs & $\begin{array}{r}0,997 \\
\mathrm{XX}\end{array}$ & $\begin{array}{r}0,564 \\
N S\end{array}$ & $\begin{array}{r}0,531 \\
\text { NS }\end{array}$ & \\
\hline
\end{tabular}

TABLEAU 5

Coefficients de corrélation entre les moyennes de divers caractères des descendances et signification statistique (cf. tabl. 2).

Correlation coefficients between various progeny characters.

\begin{tabular}{|c|c|c|c|c|}
\hline $\begin{array}{l}\text { Serre } \\
\text { chaude }\end{array}$ & $\begin{array}{c}\text { Délai } \\
\text { plantation } \\
\text { floraison }\end{array}$ & Hauteur & $\begin{array}{c}\text { Longueur } \\
\text { épi }\end{array}$ & $\begin{array}{l}\text { Nombre } \\
\text { de fleurs }\end{array}$ \\
\hline Délai plantation & & 0,492 & 0,149 & 0,819 \\
\hline floraison & & NS & NS & $\mathrm{xx}$ \\
\hline \multirow{2}{*}{ Hauteur } & 0,812 & & 0,707 & 0,645 \\
\hline & $\mathrm{xx}$ & & $\mathrm{xx}$ & $x x$ \\
\hline \multirow{2}{*}{ Longueur épi } & 0,464 & 0,580 & & 0,509 \\
\hline & NS & $\mathrm{x}$ & & NS \\
\hline \multirow{2}{*}{ Nombre de fleurs } & 0,860 & 0,666 & 0,492 & \\
\hline & $\mathrm{xx}$ & $\mathrm{xX}$ & NS & \\
\hline
\end{tabular}

TABLEAU 6

Coefficients de corrélation entre la précocité de floraison et le nombre de fleurs par épi chez 8 descendances à floraison précoce. Signification statistique (cf. tabl. 2).

Correlation coefficients between flowering earliness and spike floret number for 8 early-flowering progenies.

\begin{tabular}{ccccc}
\hline \hline Croisement & \multicolumn{2}{c}{ Serre chaude } & \multicolumn{2}{c}{ Serre froide } \\
\hline 34 & 0,11 & NS & $-0,06$ & NS \\
46 & 0,35 & $\mathrm{X}$ & 0,09 & $\mathrm{NS}$ \\
45 & 0,25 & $\mathrm{NS}$ & $-0,06$ & $\mathrm{NS}$ \\
14 & 0,06 & $\mathrm{NS}$ & 0,04 & $\mathrm{NS}$ \\
35 & 0,21 & $\mathrm{NS}$ & $-0,04$ & $\mathrm{NS}$ \\
36 & 0,10 & $\mathrm{NS}$ & 0,01 & $\mathrm{NS}$ \\
24 & 0,08 & $\mathrm{NS}$ & $-0,06$ & $\mathrm{NS}$ \\
56 & 0,18 & $\mathrm{NS}$ & $-0,02$ & $\mathrm{NS}$ \\
\hline \hline
\end{tabular}

cas sur 16; il est donc possible de sélectionner des individus à floraison précoce et possédant un nombre élevé de fleurs par épi.

\section{B. Valeur en croisement des clones parentaux}

Les conclusions de l'analyse de variance du dispositif expérimental et du plan de croisement pour les descendances et ce pour chaque caractère sont regroupées dans le tableau 7. L'effet serre est significatif pour les 4 premiers caractères. L'aptitude générale à la combinaison est la source majeure de variation. L'aptitude spécifique à la combinaison n'est significative que pour le nombre de fleurs par épi et pour la valeur esthétique de l'épi.

Les valeurs de l'aptitude générale à la combinaison des clones parentaux pour les divers caractères, ainsi que leur signification statistique, sont portées sur le tableau 8. Ce tableau illustre la valeur des clones en intercroisement; par exemple, les clones 3 et 4 sont des géniteurs de précocité, donnant en moyenne des plantes basses à épi court possédant peu de fleurs et ayant une faible valeur ornementale.

\section{Calcul des coefficients de régression}

Ces coefficients et leur intervalle de confiance au seuil 5 p. 100 ont été calculés pour les 6 caractères sous les 2 conditions (tabl. 9). Deux groupes de caractères sont à distinguer :

- la date de floraison, la hauteur de la plante, le nombre de fleurs par épi et dans une moindre mesure la longueur de l'épi ont des coefficients de régression parent moyen-descendance de valeur élevée. Le cas de la régression de la moyenne des précocités de floraison des descendances selon la précocité de floraison du parent moyen en serre froide est illustré sur la figure 1 ;

- l'esthétique de l'inflorescence et la valeur globale de la plante ont par contre des coefficients non significativement différents de zéro, c'est-à-dire que la

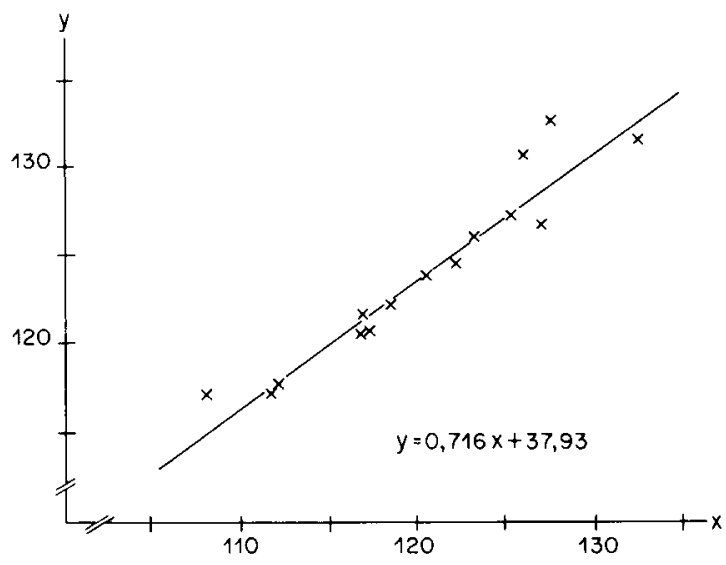

Figure 1

Régression en serre froide de la précocité moyenne de floraison des descendances selon la précocité de floraison du parent moyen. $x=$ Nombre de jours entre la plantation et la floraison chez les parents moyens.

$y=$ Nombre moyen de jours entre la plantation et la floraison chez les descendances.

Regression, in cold greenhouse, of the mean flowering earliness of progenies on the mean value of the parents. 
TABLEAU 7

Analyse de variance des valeurs en croisement pour 6 caractères. Signification statistique (Si) et contribution à la variation totale (\% SCE). Analysis of variance of breeding value for 6 characters. Levels of significance (Si) and contribution to total variation (\% SCE).

\begin{tabular}{|c|c|c|c|c|c|c|c|c|c|c|c|c|}
\hline \multirow[b]{2}{*}{ Source de variation } & \multicolumn{2}{|c|}{$\begin{array}{l}\text { Délai plantation } \\
\text { floraison }\end{array}$} & \multicolumn{2}{|c|}{$\begin{array}{l}\text { Hauteur } \\
\text { plante }\end{array}$} & \multicolumn{2}{|c|}{$\begin{array}{l}\text { Longueur } \\
\text { épi }\end{array}$} & \multicolumn{2}{|c|}{$\begin{array}{l}\text { Nombre de } \\
\text { fleurs }\end{array}$} & \multicolumn{2}{|c|}{$\begin{array}{l}\text { Esthétique } \\
\text { épi }\end{array}$} & \multicolumn{2}{|c|}{$\begin{array}{l}\text { Valeur } \\
\text { globale }\end{array}$} \\
\hline & $\mathrm{Si}$ & $\% \mathrm{SCE}$ & $\mathrm{Si}$ & $\% \mathrm{SCE}$ & $\mathrm{Si}$ & $\% \mathrm{SCE}$ & $\mathrm{Si}$ & $\% \mathrm{SCE}$ & $\mathrm{Si}$ & $\% \mathrm{SCE}$ & $\mathrm{Si}$ & $\% \mathrm{SCE}$ \\
\hline Serre & $\mathrm{xxx}$ & 7,7 & $\mathrm{xxx}$ & 29,4 & $\mathrm{xxx}$ & 11,0 & $\mathrm{xx}$ & 2,4 & & 0,1 & & 0,4 \\
\hline Répétition par serre & & 0,9 & & 1,1 & & 0,0 & & 0,1 & & 0,9 & & 2,3 \\
\hline $\begin{array}{l}\text { Aptitude générale } \\
\text { à la combinaison }\end{array}$ & $\mathrm{xxx}$ & 78,4 & $\mathrm{xxx}$ & 43,7 & $\mathrm{xxx}$ & 46,5 & $\mathrm{xxx}$ & 75,7 & $\mathrm{xxx}$ & 39,5 & $\mathrm{xxx}$ & 35,0 \\
\hline $\begin{array}{l}\text { Aptitude spécifique } \\
\text { à la combinaison }\end{array}$ & & 3,0 & & 6,3 & & 12,0 & $\mathrm{xx}$ & 9,9 & $\mathrm{X}$ & 19,6 & & 17,6 \\
\hline
\end{tabular}

TABLEAU 8

Comparaison des aptifudes générales à la combinaison des clones. Signification statistique

(Test de Newman-Keuls. Deux clones suivis de la même letlre ne sont pas significativement différents l'un de l'autre au risque $5 \%$ ).

General combining ability values of the parental clones. Multiple comparison

(Newman-Keuls test. Two figures followed by the same letter are not significantly different at the $5 \%$ level).

\begin{tabular}{ccccccc}
\hline \hline Clones & $\begin{array}{c}\text { Délai plantation } \\
\text { floraison }\end{array}$ & $\begin{array}{c}\text { Hauteur } \\
\text { plante }\end{array}$ & $\begin{array}{c}\text { Longueur } \\
\text { épi }\end{array}$ & $\begin{array}{c}\text { Nombre } \\
\text { de fleurs }\end{array}$ & $\begin{array}{c}\text { Esthétique } \\
\text { épi }\end{array}$ & $\begin{array}{c}\text { Valeur } \\
\text { globale }\end{array}$ \\
\hline 1 & $4,52 \mathrm{a}$ & $6,95 \mathrm{a}$ & $0,74 \mathrm{a}$ & $0,96 \mathrm{a}$ & $-0,10 \mathrm{a}$ & $-0,09 \mathrm{a}$ \\
2 & $5,16 \mathrm{a}$ & $2,06 \mathrm{~b}$ & $0,58 \mathrm{a}$ & $1,40 \mathrm{~b}$ & $-0,08 \mathrm{a}$ & $-0,06 \mathrm{a}$ \\
3 & $-3,75 \mathrm{~b}$ & $-7,21 \mathrm{c}$ & $-1,73 \mathrm{~b}$ & $-0,55 \mathrm{c}$ & $-0,01 \mathrm{a}$ & $-0,01 \mathrm{a}$ \\
4 & $-7,62 \mathrm{c}$ & $-4,42 \mathrm{~cd}$ & $-0,65 \mathrm{ab}$ & $-1,73 \mathrm{~d}$ & $-0,10 \mathrm{a}$ & $-0,07 \mathrm{a}$ \\
5 & $1,90 \mathrm{~d}$ & $-2,26 \mathrm{~d}$ & $-2,16 \mathrm{~b}$ & $-0,17 \mathrm{ce}$ & $0,11 \mathrm{~b}$ & $0,09 \mathrm{~b}$ \\
6 & $-0,21 \mathrm{e}$ & $4,88 \mathrm{~b}$ & $3,23 \mathrm{c}$ & $0,08 \mathrm{e}$ & $0,18 \mathrm{~b}$ & $0,14 \mathrm{~b}$ \\
\hline \hline
\end{tabular}

TABLEAU 9

Coefficients de régression parent moyen-descendance pour divers caractères en serre chaude et en serre froide. Signification statistique (cf. tabl. 2).

Mid-parent-offspring regression coefficients of various characters in heated and cold greenhouse.

\begin{tabular}{|c|c|c|c|c|}
\hline Caractères & Serre chaude & Signification & Serre froide & Signification \\
\hline Délai plantation floraison & $0,841 \pm 0,212$ & $\mathrm{xx}$ & $0,716 \pm 0,134$ & $\mathbf{x x}$ \\
\hline Hauteur & $0,668 \pm 0,318$ & $\mathrm{xx}$ & $0,717 \pm 0,361$ & $\mathrm{XX}$ \\
\hline Longueur épi & $0,343 \pm 0,307$ & $\mathrm{x}$ & $0,535 \pm 0,498$ & $\mathrm{x}$ \\
\hline Nombre de fleurs & $0,664 \pm 0,198$ & $\mathrm{xx}$ & $0,954 \pm 0,279$ & $\mathrm{xx}$ \\
\hline Valeur épi & $-0,148 \pm 0,578$ & NS & $-0,083 \pm 0,592$ & NS \\
\hline Valeur plante & $0,126 \pm 0,435$ & NS & $0,236 \pm 0,508$ & NS \\
\hline
\end{tabular}

TABLEAU 10

Coefficients de corrélation de rang de Spearman entre le classement des descendances et celui du parent moyen pour divers caractères en serre chaude et en serre froide. Signification statistique (cf. tabl. 2).

Spearman range correlation coefficients between offspring and mid-parent ranges for various characters in heated and cold greenhouse.

\begin{tabular}{|c|c|c|c|c|}
\hline Caractères & Serre chaude & Signification & Serre froide & Signification \\
\hline Délai plantation floraison & 0,90 & $\mathrm{xx}$ & 0,97 & $\mathrm{xx}$ \\
\hline Hauteur & 0,81 & $\mathrm{xx}$ & 0,82 & $\mathrm{xx}$ \\
\hline Longueur épi & 0,56 & $\mathrm{x}$ & 0,59 & $\mathrm{x}$ \\
\hline Nombre de fleurs & 0,89 & $\mathrm{xx}$ & 0,85 & $\mathrm{xx}$ \\
\hline Valeur épi & $-0,19$ & NS & 0,01 & NS \\
\hline Valeur plante & $-0,02$ & NS & 0,31 & NS \\
\hline
\end{tabular}

valeur moyenne d'une descendance ne peut être prédite par la connaissance de la valeur propre des parents.

Le calcul du coefficient de corrélation $r_{s}$ de SPEARMAN (tabl. 10) confirme les résultats précédents et per- met de penser que les mesures sont distribuées normalement.

La bonne concordance entre les valeurs obtenues sous les 2 conditions de culture pour les 2 coefficients est à noter. 


\section{DISCUSSION. CONCLUSIONS}

L'analyse d'un plan de croisement diallèle, sans réciproques ni autofécondation, faisant intervenir 6 clones de glaïeul, fait apparaître la contribution prépondérante de l'aptitude générale à la combinaison à la variation observée entre descendances pour 6 caractères notés sous 2 conditions de culture.

Le calcul du coefficient de régression - considéré comme une estimation de l'héritabilité au sens étroit - chez les 15 couples parent moyen-descendance du même dispositif permet de distinguer 2 groupes parmi les 6 caractères étudiés. La précocité de floraison exprimée par le nombre de jours s'écoulant entre la plantation et la floraison, la hauteur de la plante, le nombre de fleurs par épi et dans une moindre mesure la longueur de l'épi ont une héritabilité élevée. Cette situation conduit à une possibilité de sélection efficace pour ces caractères par un choix raisonné des géniteurs. La valeur ornementale de l'inflorescence et la valeur globale de la plante ont par contre une héritabilité non différente de zéro. Pour ces caractères de qualité, la sélection sera difficile; la connaissance phénotypique des géniteurs ne permet pas de prédire la valeur moyenne de la descendance. Dans ce cas, le sélectionneur a intérêt à réaliser un grand nombre de croisements, à étudier les descendances sur un nombre limité d'individus, puis à reprendre les combinaisons les plus prometteuses avec des effectifs plus importants. Cette façon de procéder permettra également de détecter les clones possédant une bonne aptitude générale à la combinaison.

Les estimations de l'héritabilité des caractères obtenues dans ce travail ne sont applicables en toute rigueur qu'aux 6 génotypes utilisés et ce dans les conditions de culture précisées (cela est également valable pour les estimations des aptitudes générales à la combinaison des clones). Cependant, la bonne concordance des estimations sous 2 conditions différentes et l'expérience acquise avec d'autres génotypes suggèrent qu'une certaine généralisation de ces résultats est possible. Néanmoins, seules des études similaires portant sur du matériel différent peuvent permettre une telle généralisation. Les 6 clones étudiés peuvent être considérés comme représentatifs de la gamme de géniteurs utilisés dans le programme d'amélioration. Cependant, ce nombre est trop faible et pour ce type d'étude, d'autres dispositifs expérimentaux auraient pu être adoptés, par exemple un dispositif de type pyramidal privilégiant le nombre de clones parentaux au détriment du nombre de couples parent moyendescendance et donc de la précision des coefficients calculés.

Les héritabilités calculées pour les caractères de qualité sont caractérisées par leur faible valeur et leur forte imprécision. Cela tient à 2 causes principales. La faible variabilité des notes chez les clones parentaux pour ces caractères conduit à une forte imprécision du coefficient de régression. L'utilisation d'une gamme de géniteurs très différents entre eux du point de vue esthétique aurait sans doute conduit à des valeurs plus élevées de l'héritabilité ; mais en pratique, le sélectionneur n'utilisera pas une telle gamme. Une seconde cause de la faible valeur de l'héritabilité tient au fait qu'il s'agit de caractères complexes difficiles à juger dont l'appréciation globale, exprimée par une note, fait intervenir plusieurs jugements élementaires plus ou moins subjectifs. Le fractionnement de l'esthétique de l'épi et de la valeur globale de la plante en éléments plus simples devrait permettre de mieux aborder les problèmes d'héritabilité de ces caractères.

Les conclusions que l'on peut tirer de ce travail portent sur les valeurs moyennes des descendances; or le sélectionneur n'est intéressé que par les individus les plus performants. Ceux-ci ont le plus de chances d'être présents dans les familles aux moyennes les plus élevées et présentant en même temps une forte variance. Le problème de la prédiction de cette variabilité est posé.

L'hétérogénéité phénotypique observée chez les clones parentaux et mesurée par les écarts-types est relativement forte. Cette hétérogénéité peut partiellement s'expliquer par l'accumulation de petites différences physiologiques et sanitaires entre cormus d'un même clone. Cette observation conduit à être prudent quant à la valeur du jugement porté sur un génotype représenté par une seule plante dans le cas de caractères comme la précocité de floraison, la hauteur des plantes ou le nombre de fleurs par épi et donc à ne pas être trop sévère lors de la première sélection qui ne porte que sur un seul individu.

Une des caractéristiques de la sélection du glaïeul est le nombre élevé de caractères à prendre en compte ; une nouveauté doit posséder de nombreuses qualités recherchées par les différents opérateurs de la filière : facilité de la multiplication et du grossissement pour le producteur de bulbes, sécurité et constance de la qualité de la floraison pour l'horticulteur, aptitude à la conservation et au transport pour le distributeur, esthétique et tenue en vase pour le consommateur. Il s'agit très souvent de caractères complexes, difficiles et laborieux à noter et dont le jugement ne peut souvent intervenir qu'après plusieurs années de multiplication clonale. On comprend mieux pourquoi la majorité des très nombreuses nouveautés de glaïeul proposées chaque année dans le monde disparaît rapidement. Les premières années de commercialisation peuvent être considérées comme une fin de sélection durant laquelle seuls les génotypes exceptionnels sont retenus.

Recu le 27 avril 1987. Accepté le 20 décembre 1987.

\section{REMERCIEMENTS}

Les calculs d'analyse de variance ont été effectués à la Station d'Amélioration des Plantes Fourragères, I.N.R.A., Lusignan, grâce à l'obligeance de Y. Hebert que nous remercions. Ses remarques constructives, ainsi que celles de E. BERninger (Station d'Amélioration des Plantes Florales, I.N.R.A., Fréjus) ont été appréciées.

\section{ANNEXE 1}

Généalogie des clones utilisés comme parents Pedigree of clones used as parents

1. 72.78 .5 : Saga $\times$

[(Happy End $\times$ Carmen $) \times$ (Happy End $\times$ Turkana) $]$ 2. 73.22.6: L'Innocence $\times$ 'H. van der Mark

3. 73.65.4: (Happy End $\times$ H. van der Mark) $\times$ Saga 
4. 77.81 .2 :

[(Happy End $\times$ Turkana) $\times$ Saga]

[[(Joli Coeur $\times$ Life Flame $) \times($ Happy End $\times$ Carmen $)]$ $\times$ Gladiolus angolensis]
5. 78.25 .1 :

[(Joli Cour $\times$ Life Flame $) \times($ Happy End $\times$ Carmen $)]$

$x$ Peter Pears

6. 79.2.2 : Bacchardi $\times$ Hiberia

\section{RÉFÉRENCES BIBLIOGRAPHIQUES}

Fernandez G. C. J., Miller J. C., 1985. Estimation of heritability by parent-offspring regression. Theor. Appl. Genet., 70, 650-654.

Ohri D., Khoshoo T. N., 1985. Cytogenetical evolution of garden gladiolus. Nucleus, 28, 3, 216-221.

Paulin A., 1958. Etude de l'action de températures élevées sur les cormus de Glaïeuls en vue de hâter la floraison. Rev. Hortic., 4, 1921-1923.
Simmonds N. W., 1979. Principles of crop improvement. Longman, London and New York, $408 \mathrm{p}$.

Snedecor G. W., Cochran W. G., 1957. Méthodes statistiques (traduction française). Acta, Paris, 649 p. 